\title{
Intensivmedizinische Behandlung von Erwachsenen mit angeborenen Herzfehlern
}

\author{
Bettex, D ; Bosshart, M ; Chassot, P G ; Rudiger, A
}

\begin{abstract}
Due to improvements in cardiac surgery and perioperative care the number of adults with congenital heart disease is continuously growing. The perioperative and intensive care management of these patients is a challenge due to the variety of pathologies and surgical options as well as the complex pathophysiology. Many patients develop organ dysfunction with time and many require multiple cardiac operations as well as non-cardiac interventions during adulthood. While these patients are best treated in dedicated tertiary centers that provide a multidisciplinary expertise, basic knowledge of this population is important for everyone involved in acute medical care. This review will discuss some general aspects of adults with congenital heart disease such as pulmonary hypertension, Eisenmenger syndrome, cyanosis, pregnancy and perioperative care, with a special focus on the management of critically ill patients.
\end{abstract}

DOI: https://doi.org/10.1007/s00063-012-0139-5

Other titles: Intensive care management of critically ill adults with congenital heart disease

Posted at the Zurich Open Repository and Archive, University of Zurich

ZORA URL: https://doi.org/10.5167/uzh-84295

Journal Article

Published Version

Originally published at:

Bettex, D; Bosshart, M; Chassot, P G; Rudiger, A (2013). Intensivmedizinische Behandlung von Erwachsenen mit angeborenen Herzfehlern. Medizinische Klinik, 108:561-568.

DOI: https://doi.org/10.1007/s00063-012-0139-5 


\section{Leitthema}

Med Klin Intensivmed Notfmed 2013.

108:561-568

DOI 10.1007/s00063-012-0139-5

Eingegangen: 14. Juni 2013

Angenommen: 29. Juli 2013

Online publiziert: 29. August 2013

(c) Springer-Verlag Berlin Heidelberg 2013

Redaktion

A. Geppert, Wien

G. Heinz, Wien

D. Bettex ${ }^{1}$ - M. Bosshart ${ }^{1}$ - P.G. Chassot ${ }^{2}$ - A. Rudiger ${ }^{1}$

${ }^{1}$ Kardioanästhesie und Intensivmedizin, Institut für Anästhesiologie, Universitätsspital Zürich

${ }^{2}$ Institut für Anästhesiologie, Universitätsspital Lausanne

\section{Intensivmedizinische} Behandlung von Erwachsenen
mit angeborenen Herzfehlern

ßer ist als die pädiatrische AHF-Population $[13,65]$. Die Hospitalisierungsrate ist für Patienten mit AHF doppelt so hoch wie in der normalen Bevölkerung [37, 63]. Von den AHF-Patienten, die sich auf der Notfallstation vorstellen, sterben $20 \%$ oder benötigen eine Transplantation innerhalb von 3 Jahren [26].

Die Gruppe der komplexen AHFPatienten ohne Korrektur kann unter Langzeitfolgen der chronischen Hypoxie und des pathologischen Lungenblutflusses (LBF) leiden ( $\bullet$ Infobox 1$)$. Neben langfristigen kardialen Komplikationen können auch nichtkardiale Folgen, wie

- sekundäre Erythrozytose,

- Cholelithiasis,

- Nephrolithiasis,

- Entwicklungsanomalien,

- Anomalien des zentralen Nervensystems (z. B. Krampfanfälle) aufgrund von früheren thromboembolischen Ereignissen,

- Verlust des Hör- oder Sehsinns sowie

- Lungenerkrankungen,

auftreten $[8,28]$.

Angeborene Herzfehler kommen bei fast $1 \%$ der Lebendgeburten vor, von denen ungefähr 1 von 1000 Kindern mit komplexen Missbildungen geboren wird (• Tab. 1, [40, 65]). Vor 60 Jahren haben nur $20 \%$ der Betroffenen überhaupt das Erwachsenenalter erreicht [38]. Dank Fortschritten in der Herzchirurgie und der perioperativen Betreuung überleben heutzutage über 90\% der Kinder mit AHF, was zu einer Prävalenz von ungefähr 4 von 1000 Erwachsenen geführt hat $[40,55]$. Dies bedeutet ein stetiges Wachstum dieser neuen Patientengruppe, die mittlerweile grö-

\section{Arrhythmien}

Arrhythmien sind der Hauptgrund für die Hospitalisierung von AHF-Patienten [59] und machen über die Hälfte aller Notaufnahmen aus [26]. Zur deren Entwicklung tragen

- die angeborenen Anomalien selbst,

- hämodynamische Verschlechterungen,

- atriale und ventrikuläre Dilatation,

- Fibrose und

- chirurgische Narben bei [13]. In einer retrospektiven Studie hatten 18\% der AHF-Patienten supraventrikuläre Arrhythmien, wobei die höchste Inzidenz bei der Fontan-Gruppe (45\%) beobachtet wurde. Das Vorkommen von ventrikulären Arrhythmien betrug 5\% im Allgemeinen und 14\% in der Fallot-Tetralogie(ToF)-Gruppe [16]. Vorhofflattern tritt häufig bei

- Vorhofseptumdefekt (ASD),

- Ebstein-Anomalie,

- Senning-Eingriffen,

- ToF,

- korrigierter Transposition der großen Gefäße (TGA) oder nach

- Fontan-Eingriffen

auf und birgt die Gefahr des plötzlichen Tods in sich, wenn die ventrikuläre Überleitung 1:1 beträgt [32].

Das Auftreten von Arrhythmien ist ein ungünstiges prognostisches Zeichen.

Der Verlust der atrioventrikulären Synchronie und eine schlechte Frequenzkontrolle können das Herzzeitvolumen $(\mathrm{HZV})$ erheblich beeinträchtigen. Eine

Tab. 1 Relative Häufigkeit von angeborenen Herzfehlern. (Adaptiert nach [65])

\begin{tabular}{|ll|}
\hline Azyanotische Läsionen & \\
\hline - Ventrikelseptumdefekt & $35 \%$ \\
\hline - Vorhofseptumdefekt & $9 \%$ \\
\hline - Persistierender Ductus arteriosus & $8 \%$ \\
\hline - Pulmonalstenose & $8 \%$ \\
\hline - Koarktation der Aorta & $6 \%$ \\
\hline - Aortenstenose & $6 \%$ \\
\hline - Atrioventrikulärer Kanal & $3 \%$ \\
\hline Zyanotische Läsionen & \\
\hline - Fallot-Tetralogie & $5 \%$ \\
\hline - Transposition der großen Arterien & $4 \%$ \\
\hline
\end{tabular}


Infobox 1 Gründe für die Überweisung an ein Zentrum für angeborene Herzfehler (adaptiert nach [31])

- Erhebliche intrakardiale Shunts

- Mehr als leicht erhöhter pulmonaler Gefäßwiderstand

- Mehr als mittelschwere linksventrikuläre Dysfunktion

- Mehr als leichte rechtsventrikuläre Dysfunktion

- Systemische rechte Herzkammer

- Funktionell univentrikuläres Herz

- Mehr als leichte obstruktive Herzklappenoder Gefäßerkrankungen (außer isolierte Aortenstenose und viele Fälle von isolierten Mitralklappenerkrankungen)

- Erhebliche angeborene Anomalien der Koronararterien

- Schwangerschaft bei Patientinnen mit schwerem angeborenem Herzfehler

- Neues Auftreten von symptomatischen Tachy- oder Bradykardien

Elektrokonversion in den Sinusrhythmus sollte erst nach Ausschluss eines intraatrialen Thrombus mittels transösophagealer Echokardiographie (TOE) erfolgen. Auch wenn die Anästhesie bei einer Kardioversion relativ unkompliziert ist, sollte ein Notfallplan vorhanden sein, falls der Eingriff zu einer Bradykardie führt. Dies ist v. a. bei Patienten ohne intravenösem Zugang zum Ventrikel wichtig. Die akute intraatrialen Reentrytachykardie (IART) wird primär durch Adenosin behandelt $[5,45]$. Seine Vorteile sind die kurze Halbwertzeit und das Fehlen von negativ inotroper Wirkung. Wegen der hohen Rezidivrate ist eine Prophylaxe mit Digoxin, Verapamil oder Klasse-1C-Medikamenten, wie Flecainide, Propafenon oder Amiodaron, nötig. Die pharmakologische Behandlung einer IART bleibt aber langzeitig meist erfolglos. Die kurative Vorgehensweise ist die Radiofrequenzablation [27]. Amiodaron kann aufgrund von schweren nichtkardialen unerwünschten Wirkungen v. a. bei jungen Patienten nur eingeschränkt verabreicht werden [61].

Die Behandlung einer Bradykardie, der zweithäufigsten Arrhythmie, erfordert häufig einen Herzschrittmacher. Eine Dysfunktion des Sinusknotens tritt beispielsweise bei bis zu 40\% der FontanPatienten auf [42]. Allerdings stellen feh- lende Venenzugänge zum Herzen, Elektroden in nicht standardisierten Kammern, hohe Reizschwellen, niedrige Reizempfindlichkeit, Infektionen und Dyslokation der Elektroden potentielle Probleme dar [50, 58]. Patienten mit einem Rechts(R)-links(L)-Shunt müssen aufgrund des Risikos einer systemischen Thromboembolie antikoaguliert werden.

\section{1) Das Auftreten von Arrhythmien ist ein ungünstiges prognostisches Zeichen}

Ventrikuläre Arrhythmien sind seltener und treten meist aufgrund von Läsionen des rechtsventrikulären Ausflusstrakts (RVOT) bei ToF oder Pulmonalatresie auf. Andere Risikofaktoren sind:

- schlechte Ventrikelfunktion,

- vorangegangene Ventrikulostomie,

- hohes Alter beim Ersteingriff [8],

- QRS-Verlängerung über $180 \mathrm{~ms}$, v. a. in Verbindung mit einer pulmonalen Regurgitation (PR) und rechtsventrikulärer (RV) Dilatation [22].

Eine Ablation hat unterschiedliche Erfolgsaussichten, und eine antiarrhythmische Behandlung konnte nicht mit einer verbesserten Überlebensdauer in Verbindung gebracht werden. Interne Defibrillatoren werden immer häufiger implantiert, obwohl die Indikationen weniger klar definiert sind als bei Erwachsenen ohne AHF $[18,46]$. Elektrodendysfunktionen sind häufig, und die Funktionsdauer der Elektroden beträgt nach 8 Jahren lediglich 60\% [29]. Diese hohe Dysfunktionsrate bedingt gerade bei jungen Patienten mit einer Lebenserwartung von mehreren Dekaden wiederholte operative Eingriffe und ein erhöhtes Risiko von entsprechenden Komplikationen [4].

Der plötzliche Herztod war die häufigste Todesursache (26\%) in einer Querschnittstudie mit AHF-Patienten [44], wobei ToF, TGA, Koarktation der Aorta, Aortenstenose (AS) und schwere linksventrikuläre (LV) Dysfunktion die höchsten Risiken waren.

\section{Infektiöse Endokarditis}

Die infektiöse Endokarditis liegt etwa 5\% der AHF-Einweisungen zugrunde. Kleine Ventrikelseptumdefekte (VSD) und Anomalien des linksventrikulären Ausflusstrakts (LVOT) sind dabei die am häufigsten vorkommenden Läsionen [33]. Bei einem nichtkorrigierten VSD beträgt das Risiko für das Auftreten einer infektiösen Endokarditis während der Lebensdauer $10-15 \%$. Da die transthorakale Echokardiographie nur bei $60 \%$ der Betroffenen zu einem positiven Befund führt, ist die TOE meistens indiziert. Die Magnetresonanztomographie kann in Fällen von falschen Aneurysmen, infizierten Shunts oder Conduits vorgezogen werden. Die Endokarditisprophylaxe ist indiziert bei Patienten,

- die bereits eine Endokarditis hatten,

- mit nichtkorrigierten zyanotischen AHF,

- bis zu 6 Monate nach der Implantation von Fremdmaterial und

- mit zurückgebliebenen Defekten an einer Patch- oder Implantatstelle [24].

\section{Herzinsuffizienz}

Eine verschlechterte Pumpfunktion, v. a. rechtsseitig, tritt bei Patienten mit AHF häufig auf. Dabei ist das RV-Versagen ein entscheidender Faktor bei der Beurteilung des klinischen Status und der Langzeitprognose [12, 64]. Im Allgemeinen wird die RV-Druckbelastung weniger gut toleriert als die Volumenbelastung. Bei zyanotischen Patienten stellt sich das RVVersagen meist nach dem 40. Lebensjahr ein. Ihnen kann mit Angiotensin-converting-enzyme(ACE)-Hemmern nicht geholfen werden, da diese Medikamente die Zyanose noch verschlimmern. Aufgrund der zahlreichen Voroperationen und den meist komplexen Antikörperkonstellationen gehen Transplantationen mit Schwierigkeiten und schlechteren Ergebnissen einher.

Die intensivmedizinische Behandlung der RV-Dysfunktion beinhaltet [47, 52, 53]:

- Optimierung der Vorlast (Volumenentzug bei RV-Dilatation), 
- Erhöhung des systemischen Blutdrucks, um die Koronarperfusion zu sichern,

- Steigerung der Inotropie (Dobutamin, Adrenalin, Milrinon, Levosimendan),

- Herzfrequenz- und Rhythmuskontrolle (atriovenrtikuläre (AV) Synchronisation),

- Senkung der RV-Nachlast mittels guter Oxygenierung, Vermeiden von Hyperkapnie, Azidose und exzessiven Beatmungsdrücken sowie Einsatz von pulmonalen Vasodilatatoren (Stickstoffmonoxid, inhalierte Prostaglandin);

- Evaluation von mechanischen Unterstützungssystemen, wie die intraaortale Ballonpumpe oder extrakorporale Membranoxygenierung (ECMO).

\section{Zyanose}

Eine Zyanose (Desoxyhämoglobin $>5 \mathrm{~g} /$ dl) entsteht aufgrund eines unzureichenden LBF in Zusammenhang mit einem RL-Shunt, kann aber auch aufgrund eines übermäßigen LBF bei einem L-R-Shunt auftreten, wenn der Shunt durch ein Stealphänomen zu einer systemischen Hypoperfusion führt [35]. Tritt eine verringerte Systemperfusion auf, müssen

- eine Dehydration vermieden,

- der systemische Gefäßwiderstand (SVR) aufrechterhalten,

- der pulmonale Gefäßwiderstand (PVR) erhöht und

- der Sauerstoffverbrauch minimiert werden.

Das beste hämodynamische Gleichgewicht wird erreicht, wenn der pulmonale Fluss (Qp) möglichst nahe beim systemischen Fluss (Qs) liegt.

Patienten mit einer Ruhesättigung unter $85 \%$ bei Raumluft müssen einen Hämoglobinwert größer als $180 \mathrm{~g} / \mathrm{l}$ haben, um eine Hypoxämie auszugleichen [2]. Allerdings führt eine Polyzythämie zu einer Hyperviskosität, welche die Mikrozirkulation beeinträchtigt und das Risiko einer Thromboembolie erhöht [43]. Eine chronische Hypoxie kann ebenfalls Herzmuskel- und Nierenfunktionsstörungen verschlimmern [19].

Wird kein palliatives Verfahren zur Erhöhung des LBF eingeleitet, können

Med Klin Intensivmed Notfmed 2013 • 108:561-568 DOI 10.1007/s00063-012-0139-5

(c) Springer-Verlag Berlin Heidelberg 2013

\section{Bettex · M. Bosshart · P.G. Chassot · A. Rudiger Intensivmedizinische Behandlung von Erwachsenen mit angeborenen Herzfehlern}

\section{Zusammenfassung}

Dank Verbesserungen in der Herzchirurgie und der perioperativen Betreuung steigt die Anzahl der Erwachsenen mit einem angeborenen Herzfehler stetig. Die perioperative und intensivmedizinische Betreuung dieser Patienten stellt aufgrund der vielen verschiedenen Pathologien und chirurgischen Möglichkeiten sowie der komplexen Pathophysiologie eine Herausforderung dar. Viele Patienten entwickeln mit der Zeit Organdysfunktionen und viele von innen benötigen Folgeoperationen am Herzen sowie nichtkardiale Eingriffe im Erwachsenalter. Aufgrund der Komplexität sind diese Patienten in spezialisierten tertiären Krankenhäusern zu behandeln, die über ein disziplinenübergreifen- des Fachwissen verfügen. Da dies in Notfällen nicht immer möglich sein wird, sind grundlegende Kenntnisse dieser Patientengruppe für in der Akutversorgung Tätige wichtig. In dieser Übersicht werden allgemeine Aspekte, wie pulmonale Hypertonie, EisenmengerSyndrom, Zyanose, Schwangerschaft und perioperative Betreuung, behandelt. Ein besonderes Augenmerk gilt dabei der intensivmedizinischen Versorgung von kritisch kranken Patienten mit angeborenen Herzfehlern.

Schlüsselwörter

Perioperative Behandlung .

Eisenmenger-Syndrom - Zyanose .

Physiologie · Pulmonale Hypertonie

\section{Intensive care management of critically ill adults with congenital heart disease}

\section{Abstract}

Due to improvements in cardiac surgery and perioperative care the number of adults with congenital heart disease is continuously growing. The perioperative and intensive care management of these patients is a challenge due to the variety of pathologies and surgical options as well as the complex pathophysiology. Many patients develop organ dysfunction with time and many require multiple cardiac operations as well as non-cardiac interventions during adulthood. While these patients are best treated in dedicated tertiary centers that provide a multidisciplinary expertise, basic knowledge of this population is important for everyone involved in acute medical care. This review will discuss some general aspects of adults with congenital heart disease such as pulmonary hypertension, Eisenmenger syndrome, cyanosis, pregnancy and perioperative care, with a special focus on the management of critically ill patients.

\section{Keywords}

Perioperative care $\cdot$ Eisenmenger syndrome . Cyanosis · Physiology · Pulmonary

hypertension zyanotische Patienten erhebliche aortopulmonale Kollaterale entwickeln, aufgrund derer L-R-Shunts zusätzlich zum intrakardialen R-L-Shunt entstehen. Diese Kollateralen konkurrieren mit dem diastolischen Blutfluss in den Koronararterien und beeinträchtigen damit die Herzmuskelperfusion und -funktion.

Patienten mit zyanotischen Läsionen weisen eine abgeschwächte hypoxische Atemantwort auf, während die Antwort auf Hyperkapnie sowie auf Opioide erhalten bleibt [7].

\section{Pulmonale Hypertonie}

Die pulmonale Hypertonie (PHT) ist durch einen mittleren pulmonalarteriellen Druck (PAD) größer als $25 \mathrm{mmHg}$ definiert und schränkt die funktionelle Kapazität und die Überlebenschance von AHF-Patienten ein [17, 34, 36]. Mögliche Ätiologien sind ein erhöhter ventrikulärer enddiastolischer Druck, ein erhöhter linker Vorhofdruck oder eine Pulmonalvenenstenose. Die Hauptursache ist allerdings das Vorhandensein von langdauernden und großen L-R-Shunts. Diese verursachen einen erhöhten Fluss und eine Übertragung des fast systemischen Drucks auf das Lungenkapillarbett, was 
zu einem irreversiblen vaskulären Umbau führt [8]. Die reaktive Form der PHT zeichnet sich durch eine übertriebene Vasokonstriktion auf sympathische Stimuli (z. B. Schmerzen), Azidose, Hyperkapnie, Hypoxie und Hypothermie aus. Einer Verschlimmerung der PHT kann durch einen hohen inspiratorischen Sauerstoffanteil $\left(\mathrm{F}_{\mathrm{I}} \mathrm{O}_{2}\right)$, einen arteriellen Kohlendioxidpartialdruck $\left(\mathrm{PaCO}_{2}\right)$ zwischen 30 und $35 \mathrm{mmHg}$ und durch Sedation entgegen gewirkt werden. Eine inotrope Unterstützung in Verbindung mit einer systemischen Vasokonstriktion erhöht den Koronarperfusionsdruck und die ventrikuläre Funktion und verringert den R-L-Shunt. Die selektive pulmonale Vasodilatation mit eingeatmetem Stickstoffmonoxid ist dabei der Hauptpfeiler der Therapie, obwohl auch inhalative Prostanoide erfolgreich eingesetzt wurden. Die orale Verabreichung von Sildenafil, einem selektiven Hemmer der cGMP-spezifischen Phosphodiesterase vom Typ 5, wird prophylaktisch und therapeutisch eingesetzt [55].

\section{Eisenmenger-Syndrom}

Das Eisenmenger-Syndrom ist durch einen PVR größer als 800 dynes $\bullet$ s $\bullet \mathrm{cm}^{-5}$ und einen bidirektionalen Shuntfluss gekennzeichnet und ist die häufigste Ursache von zyanotischen Herzbeschwerden bei Erwachsenen [67]. Der zugrundeliegende Mechanismus umfasst einen progressiven vaskulären Umbau der Lungenarterien. Viele Patienten mit Eisenmenger-Syndrom erreichen ein mittleres Überleben bis in die Mitte der 3. Lebensdekade. Ein Abfall des systemischen Blutdrucks erhöht die Wahrscheinlichkeit von R-L-Shunts und verschlimmert die Zyanose, was mit kardiovaskulärem Kollaps und Todesfällen in Verbindung gebracht wird. Ein plötzlicher Anstieg des PVR führt zu einem akuten RV-Versagen und verschlimmert den R-L-Shunt. Symptomatisches RV-Versagen, Arrhythmien und alle akuten Änderungen der Vor- oder Nachlast gehen mit einer hohen Morbidität und Mortalität einher [3, 11].

Bei Patienten mit Eisenmenger-Syndrom und zyanotischen Patienten besteht das Risiko von perioperativen Blutungen und Thrombosen neben komplexen Anomalien des Koagulationssystems, wie
Thrombozytopenie und niedrige Werte für die Vitamin-K- abhängigen Gerinnungsfaktoren, den Faktor $\mathrm{V}$ und den von-Willebrand-Faktor $[8,60]$. Die Blutungsdauer ist allerdings meist aufgrund einer erhöhten Blutviskosität und eines verringerten Fluss normal. Die Erythrozytose kann die Labortests beeinträchtigen, die zur Messung von ,international normalized ratio" (INR) und der aktivierten partiellen Thromboplastinzeit verwendet werden. Dies erschwert die orale Antikoagulation, die für Patienten mit PHT indiziert ist [11].

\section{Cavopulmonale Anastomosen}

Chirurgische cavopulmonale Anastomosen sind in Fällen von ventrikulären Hypoplasien indiziert oder wenn die Wiederherstellung einer biventrikulären Zirkulation nicht möglich ist. Das palliative Verfahren leitet Blut an die Lungenarterien oder an einen Ventrikel weiter. Der bidirektionale Glenn-Shunt ist die Verbindung der Vena cava superior (VCS) mit der rechten Lungenarterie. Dadurch werden die arterielle Sättigung und die Belastungstoleranz verbessert, obwohl die Zyanose bestehen bleibt, da sich das Blut der Vena cava inferior (VCI) auf atrialer Ebene weiterhin vermischt. Die Vorteile lassen oft mit der Zeit nach, da es zu einer Entwicklung von pulmonalen arteriovenösen Fehlbildungen kommt, die zu einer Hypoxämie führen. Allerdings bestehen langfristige Überlebenschancen [30]. Der Glenn-Shunt wird normalerweise als Vorstufe vor einem Fontan-Eingriff durchgeführt, mit dem die VCI über einen intraoder extrakardialen Kanal in die Lungenarterien umgeleitet wird und das rechte Herz umgeht. Der Erhalt des rechten Vorhofs im Kreislauf hat zu einer massiven atrialen Dilation und einem erhöhten Risiko für Thromboembolien und Arrhythmien geführt.

Die Fontan-Zirkulation behebt die Mischung von systemischem und pulmonalvenösem Blut und verbessert die Zyanose. Die serielle Zirkulation entlastet auch die Volumenlast auf den Ventrikel. Allerdings sind die physiologischen Auswirkungen einer Zirkulation nach Fontan extrem [25]: Der pulmonale Fluss wird vollständig passiv gesteuert und ist ausschließlich vom Druckgradienten zwischen dem zentralen Venendruck (ZVD) und dem linksatrialen Druck (LAD) abhängig. Es wird allgemein davon ausgegangen, dass ein Gradient von 8-10 mmHg für diesen Fluss notwendig ist. Faktoren zur Erhöhung des PVR reduzieren den LBF, was zu einer inadäquaten Füllung des systemischen Ventrikels und einer Reduzierung des HZV führt. Die venöse Hypertonie ist dabei eine unausweichliche Folge des nicht existierenden RV und geht mit peripheren Ödemen einher. Die Dysfunktion des systemischen Ventrikels führt zu einem Anstieg des LAD und einer Reduzierung des LBF. Die Fenestrierung des FontanKreislaufs (Öffnung zwischen dem Conduit und dem rechten Vorhof) wird oft bei Risikopatienten eingesetzt. Sie erlaubt den Erhalt des systemischen HZV auf Kosten einer Entsättigung.

Bei einer passiven Lungenzirkulation wird eine Hypovolämie schlecht vertragen.

\section{1) Bei einer passiven \\ Lungenzirkulation wird eine Hypovolämie schlecht vertragen}

Der Blutfluss durch die venopulmonale Anastomose hängt auch zu einem Großteil von der Atmung ab. Die Vorteile der spontanen Atmung in dieser Gruppe sind hinreichend bekannt. Die Herausforderung während der Sedation besteht darin, eine Atemdepression zu verhindern und gleichzeitig eine wirksame Schmerzfreiheit zu gewährleisten. Eine erfolgreiche Fontan-Zirkulation führt zu einer arteriellen Sauerstoffsättigung $\left(\mathrm{SpO}_{2}\right)$ von mehr als $94 \%$ bei Raumluft. Bei einer Sättigung unter $90 \%$ sollte eine Überweisung an ein AHF-Zentrum zur weiteren Abklärung erfolgen.

Die Fontan-Zirkulation kann zu einer hepatischen Dysfunktion mit Cholestase und Veränderungen des Koagulationssystems führen [62]. Thromboembolien, einschließlich Lungenembolien, treten in 18\% der Fälle auf [10]. Systemische Thromboembolien und Schlaganfälle können aufgrund einer Fontan-Fenestrierung oder eines zurückgebliebenen ASD auftreten oder von einem Stumpf der Lungenarte- 


\section{Infobox 2 Kontraindikationen für eine Schwangerschaft mit einem Mortalitätsrisiko der Mutter größer als 10\% (adaptiert nach [21]) \\ - Pulmonalarterielle Hypertonie jeglicher Ursache \\ - Marfan-Syndrom mit erweiteter Aortenwurzel größer als $4 \mathrm{~cm}$ \\ - Aortendilatation größer als $5 \mathrm{~cm}$ in Verbindung mit einer bikuspiden Aortenklappe \\ - Schwere obstruktive Läsionen des linken Herzes (schwere Mitralstenose, Aorten- stenose oder Koarktation) \\ - Schwere systemisch ventrikuläre Dys- funktion (LV-Auswurffraktion kleiner als $30 \%$, New York Heart Association (NYHA) III-IV) \\ - Vorherige Peripartumkardiomyopathie mit residueller Beeinträchtigung der linksventrikulären Funktion}

\section{Infobox 3 Prädikatoren von mütterlichen kardiovaskulären Ereignissen (adaptiert nach [57]) \\ - NYHA-Funktionsklasse-III und -IV \\ - Zyanose $\left(\mathrm{SpO}_{2}\right.$ bei Raumluft kleiner als 90\%) \\ - Früheres kardiovaskuläres Ereignis \\ - Systemisch ventrikuläre Auswurffraktion kleiner als $40 \%$ \\ - Linksherzobstruktion: Mitralklappen- stenose mit einer Öffnungsfläche kleiner als $2 \mathrm{~cm}^{2}$, Aortenklappenstenose mit einer Öffnungsfläche kleiner als $1,5 \mathrm{~cm}^{2}$, Gradient im linksventrikulären Ausfluss- trakt größer als $30 \mathrm{mmHg}$}

Für jeden vorhandenen Prädikator nach Cardiac-Disease-in-Pregnancy(CARPREG)-Score wird ein Punkt vergeben. Risikobeurteilung von kardiovaskulären Komplikationen bei der Mutter:

- 0 Punkte: $5 \%$

- 1 Punkt: $27 \%$

- Mehr als 2 Punkte: $75 \%$.

rie bzw. einer systemischen Kammer herrühren.

Eine unangenehme Langzeitkomplikation ist das Auftreten des Eiweißverlustsyndroms, das zu Durchfall und Hypoproteinämie führt. Diese Patienten haben eine 50\%ige 5-Jahres-Mortalität [55]. Das Syndrom reagiert schlecht auf Diuretika, sodass eine chirurgische Korrektur des obstruierten Conduits nötig sein kann.

\section{Palliative Shunts}

Palliative Shunts werden zur Erhöhung des LBF eingesetzt, wenn eine endgültige Korrektur nicht möglich ist. Der meistverwendete Shunt ist die Verbindung zwischen der Subklaviaarterie und der Lungenarterie (Blalock-Taussig-Shunt). Der Fluss wird dabei zum Großteil von der Abmessung des Shunts und hämodynamischen Variablen bestimmt. Dadurch kann eine Senkung des systemischen vaskulären Widerstandes den LBF bei diesen Patienten dramatisch verringern.

\section{Schwangerschaft}

Wie kürzlich berichtet ist eine spezialisierte Versorgung der AHF-Patientinnen während der Schwangerschaft vorgeschrieben [21]. Ein AHF ist die häufigste Todesursache bei Müttern mit einer Herzerkrankung (• Infobox 2). Das Auftreten von Komplikationen bei schwangeren Frauen mit AHF betrug 13\%; dies schließt Lungenödeme, Arrhythmien, Lungenhochdruckkrisen, thromboembolische Komplikationen und Todesfälle ein (- Infobox 3, [57]). Im Allgemeinen werden Läsionen mit Regurgitationen besser toleriert als Stenosen. Die vaginale Entbindung ist generell indiziert, außer bei Patientinnen, die Warfarin einnehmen, an einem Marfan-Syndrom, einem Aortenaneurysma oder einer kritischen AHF leiden [21].

Erreicht eine Läsion während der Schwangerschaft einen kritischen Zustand, sollte eine Operation bis nach der Geburt aufgeschoben werden. Ist ein Aufschub unmöglich, liegt die beste Zeit für eine Operation zwischen der 13. und 28 . Woche [21]. Sind perkutane Interventionen nötig, sollten diese bevorzugt im 2. Trimester durchgeführt werden. Bei Herzoperationen und Einsatz der HerzLungen-Maschine ist die Mortalität der Mutter ähnlich wie bei nicht Schwangeren, aber das Risiko einer Fehlgeburt ist hoch. Eine peripartale Antibiotikaprophylaxe gegen die bakterielle Endokarditis wird in Fällen mit einer Endokarditisvorgeschichte empfohlen [24], ist aber Gegenstand von Diskussionen bei anderen schwangeren Frauen mit AHF [66].

\section{Hier steht eine Anzeige.}




\section{Herzoperationen}

Ungefähr 20\% der AHF-Einweisungen erfolgen in Verbindung mit Herzoperationen. Etwa die Hälfte dieser Patienten benötigt eine primäre Wiederherstellung, die anderen Patienten unterziehen sich Nachsorgeoperationen [58]. Obwohl die beobachtete chirurgische Mortalität bei Erwachsenen mit AHF niedrig ist (1,5$7 \%)$, treten bei $24 \%$ schwere postoperative Komplikationen auf [6]. Zeit an der Herz-Lungenmaschine, Fontan-Eingriff, präoperativer Hämatokrit, Anzahl der vorangegangenen Operationen und präoperative Herzinsuffizienz wurden mit einer hohen Morbidität in Verbindung gebracht [23], während ein abnormaler präoperativ durchgeführter Schilddrüsenfunktionstest sowie erhöhte Bilirubin- und Kreatininwerte eine erhöhte Sterblichkeit prognostizierten [48]. Im Laufe eines Lebens können zahlreiche Nachsorgeoperationen notwendig werden, bei denen Mortalität und Morbidität ab der 3. Nachsorgeoperation deutlich ansteigen [15].

\section{Perioperative Betreuung}

Detaillierte Informationen zur Anästhesie von AHF-Patienten sind in verschiedenen Übersichtsartikeln verfügbar $[8,9$, 35]. Das perioperative Risiko ist bei AHFPatienten erheblich erhöht. Dies trifft v. a. bei Patienten mit einer schlechten Funktionsklasse, PHT, Herzinsuffizienz und Zyanose zu [20]. Darum sollten Patienten mit einem mittelschweren oder komplexen AHF in einem spezialisierten Zentrum behandelt werden $[31,35,65]$.

\section{) Das perioperative Risiko ist bei Patienten mit angeborenem Herzfehler erheblich erhöht}

Bei einer präoperativen Beurteilung sollte - neben den genannten Risikofaktoren, wie PHT, Zyanose und Herzinsuffizienz - auch auf Arrhythmien, Restshunts, Klappenvitien, Aneurysmen und andere Komplikationen, wie Erythrozytose und Störungen des zentralen Nervensystems (Krampfanfälle, vorangegangene Schlaganfälle oder intrazerebrale Abszesse), geachtet werden [20].
Die perioperative Betreuung bei zyanotischen Patienten muss [55]

- den Lungenhochdruck senken, um den LBF zu verbessern,

- den SVR erhöhen, um R-L-Shunts zu verringern,

- die Normovolämie erhalten,

- den Hämatokrit optimieren und

- die dynamische RVOT-Obstruktion mit $\beta$-Blockern vermindern.

Eine Änderung der eingeatmeten Sauerstoffkonzentration hat nur geringe Auswirkungen auf den $\mathrm{SpO}_{2}$, während eine systemische Vasokonstriktion den $\mathrm{SpO}_{2}$ erhöht, indem R-L-Shunts verringert oder der Durchfluss durch die aortopulmonale Kollateralen verbessert werden. Eine präoperative Phlebotomie zur Verbesserung der chirurgischen Hämostase kann bei einem Hämatokrit größer als 65\% hilfreich sein.

Alle intravenösen Narkosemittel verringern den SVR, sodass die Dosis wichtiger als das eigentlich verabreichte Arzneimittel ist. Die Fähigkeit, das HZV als Reaktion auf Stress zu erhöhen, ist bei vielen angeborenen Läsionen herabgesetzt. Flüssigkeitsersatz soll eine Hypovolämie verhindern und muss luftfrei erfolgen. Luftfilter oder Blasenfänger sind dabei kein Ersatz für eine gründliche Entlüftung aller Schläuche und Injektionsanschlüsse. Eine Schnellinfusion mit Blasenfänger sollte bei der Infusion von großen Volumenmengen eingesetzt werden.

Während der maschinellen Beatmung steigt der PVR bei hohen intrathorakalen Drücken. Die Dauer der Einatmung hat dabei eine größere Wirkung als der inspiratorische Druck. Darum führt eine Verringerung der Inspirationszeit und eine darauffolgende Erhöhung des inspiratorischen Drucks normalerweise zu einer Maximierung des LBF.

Die postoperative Betreuung dieser Patienten sollte auf einer Intensivstation erfolgen, die auf die Betreuung von Herzpatienten spezialisiert ist, da dadurch sowohl Morbidität als auch Mortalität verringert werden [3]. Konventionelle Bewertungssysteme können die Mortalität von chirurgischen Patienten mit AHF nicht ge-nau vorhersagen, wohingegen die Mortalität mittels Acute-physiology-andChronic-Health-Evaluation(APACHE)-
Score-II bei medizinischen AHF-Patienten akkurat vorhergesagt werden kann [48].

\section{Überwachung}

Ist eine invasive Überwachung indiziert, muss die optimale Position eines Gefäßkatheters sorgfältig gewählt werden. Ein vorangegangener Blalock-TaussigShunt oder eine vorherige Wiederherstellung der Koarktation der Aorta können beispielsweise eine invasive Blutdrucküberwachung auf der kontralateralen Seite erfordern. Ein vorheriger Gefäßeingriff kann zu Thrombosen, Stenosen, kollateralen Gefäßen oder Vernarbung führen, die einen erfolgreichen Zugang und hämodynamische Messungen erschweren können. Zentrale Venenkatheter stellen bei Patienten nach einem Fontan-Eingriff ein erhebliches thromboembolisches Risiko dar, und eine diesbezügliche Indikation sollte nur in Ausnahmefällen erfolgen. Eine Überwachung des Pulmonalarteriendrucks ist damit zwar möglich, aber die Messung des transpulmonalen Gradienten erfordert einen chirurgisch implantierten atrialen Katheter.

Eine persistierende linksseitige VCS ist mit $0,5 \%$ bei gesunden Personen und $4 \%$ bei AHF-Patienten häufig [54]. In 92\% der Fälle entleert sich die linksseitige VCS über den Koronarsinus in den rechten Vorhof. Bei den restlichen 8\% entleert sie sich jedoch in den linken Vorhof und führt damit zu einem R-L-Shunt. Arrhythmie und myokardiale Ischämie aufgrund der Manipulation des Koronarsinus sind bei der Katheterisierung der linksseitigen VCS bekannt. Diese Anomalie kann auch Auswirkungen auf die korrekte Platzierung eines Pulmonalarterienkatheters oder von Herzschrittmacherelektroden haben.

Die Pulsoximetrie muss bei Patienten mit AHF zwingend durchgeführt werden. Allerdings können mit der Pulsoximetrie L-R-Shunts unerkannt bleiben. Die arterielle $\mathrm{SpO}_{2}$ kann normal erscheinen, auch wenn das systemische HZV schwer vermindert ist. In der Kapnographie wird der $\mathrm{PaCO}_{2}$ während der Ausatmung unterschätzt, wenn ein R-L-Shunt vorhanden ist.

Die Messung des HZV mit einem Pulmonalarterienkatheter wird aufgrund der schwierigen oder unmöglichen Plat- 
zierung, dem Risiko der Induktion von Arrhythmien und in Gegenwart von Shunts selten verwendet. Eine Pulskonturanalyse führt $\mathrm{zu}$ widersprüchlichen Ergebnissen und kann für diese Patientengruppe nicht empfohlen werden [39, 49]. Allerdings kann mit einer transpulmonalen Thermodilution eine verlässliche Messung des HZV und des intrathorakalen Blutvolumens, einem Marker für die Vorlast, erreicht werden.

Über TOE sind Echtzeitinformationen $\mathrm{zu}$ Ventrikelfunktion, Vorlast, Shunts und Herzklappenfunktion verfügbar, womit dieses Instrument während kardialen und nichtkardialen Eingriffe sehr wertvoll ist.

\section{Atemwegsprobleme}

Erwachsene mit einem komplexen AHF können aufgrund von hypoplastischen Lungenarterien, einseitiger Lungenhypoplasie, restriktiver Lungenerkrankungen, Skoliose oder Zwerchfelllähmungen eine herabgesetzte Lungenfunktion aufweisen [51]. Patienten mit primären LVErkrankungen können von einer positiven Druckbeatmung profitieren, weil der transmurale Druck reduziert wird [1]. Andererseits leiden Patienten mit einer primären RV-Dysfunktion unter einer positiven Druckbeatmung, weil dadurch der Druck im rechten Vorhof steigt und der venöse Rückfluss abnimmt. Durch die verminderte RV-Vorlast nimmt das Schlagvolumen und konsekutiv das HZV ab [1]. Eine zu hohe oder zu niedrige Blähung der Lunge führt zu einem Anstieg des PVR, einer erhöhten RV-Nachlast und einem verringerten LBF. Die Auswirkungen eines hohen positiven endexspiratorischen Drucks (PEEP) sind bei der FontanZirkulation oder RV-Dysfunktion besonders schädlich.

\section{Nieren- und Leberprobleme}

Unter den AHF-Patienten wiesen 41\% eine leichte und $9 \%$ eine mittelere bis schwer gestörte glomeruläre Filtrationsrate auf, was mit einem 3-fach erhöhtem Mortalitätsrisiko einhergeht [14]. Bei zyanotischen Patienten tritt eine gestörte Nierenfunktion noch häufiger auf [41]. Maßnahmen zum Schutz der Niere umfassen die sorgfältige Kontrolle der
Hämodynamik sowie die kritische Verabreichung von nephrotoxischen Wirkstoffen [1]. Patienten mit einem RV-Versagen können durch ein abdominelles Kompartmentsyndrom oder Aszites besonders gefährdet sein.

Erhöhte rechtsseitige Drücke verursachen eine Stauung in den Lebervenen, die zu einer Leberzellnekrose und schließlich einer Leberzirrhose führen kann [56]. Die Leberfunktion kann sich bei einem niedrigen HZV oder bei Hypoxie weiter verschlechtern [1].

\section{Wiederbelebungsmaßnahmen}

Patienten mit einer erheblichen AVKlappeninsuffizienz, PHT, schwerer links- oder rechtsseitiger Klappenstenose und Fontan-Zirkulation weisen keine guten Erfolge bei einer kardiopulmonalen Reanimation auf. Der passive LBF in der Fontan-Zirkulation findet nur in der Entlastungsphase der Herzdruckmassage statt, sodass eine vollständige Entlastung kritisch ist [1]. Eine mechanische Herzunterstützung durch ECMO ist bei Patienten mit AHF schwierig und wird durch Gefäßverschlüsse, Ischämie in den Extremitäten, Blutungen und Thromboembolien noch erschwert.

\section{Fazit für die Praxis}

- Die Zahl der erwachsenen Patienten mit AHF, die auf der Intensivstation behandelt werden müssen, steigt kontinuierlich an. Die perioperative und intensivmedizinische Betreuung dieser Patienten stellt aufgrund der vielen verschiedenen Pathologien und chirurgischen Möglichkeiten sowie der komplexen Pathophysiologie eine Herausforderung dar.

- Viele Patienten entwickeln mit der Zeit Organdysfunktionen, und viele von ihnen benötigen mehrere Nachsorgeoperationen am Herzen sowie nichtkardiale Eingriffe im Erwachsenalter. Für eine erfolgreiche Behandlung sind darum fundierte Kenntnisse der zugrundeliegenden Krankheit und ihrer Mechanismen erforderlich.

- Aufgrund der Komplexität sollten AHF-Patienten in spezialisierten tertiären Krankenhäusern betreut werden, in denen über ein interdisziplinäres Know-how verfügt wird. Da dies bei Notfällen nicht immer möglich sein wird, müssen alle Intensivmediziner über Grundkenntnisse in der Behandlung von AHF verfügen.

\section{Korrespondenzadresse

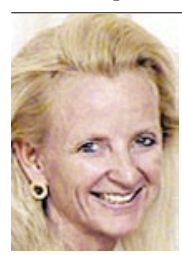 \\ PD Dr. D. Bettex \\ Kardioanästhesie und Intensivmedizin, Institut für Anästhesiologie, Universitätsspital Zürich Rämistr. 100, 8091 Zürich Schweiz dominique.bettex@usz.ch}

\section{Einhaltung ethischer Richtlinien}

Interessenkonflikt. D. Bettex, M. Bosshart, P.G. Chassot und A. Rudiger geben an, dass kein Interessenkonflikt besteht.

Dieser Beitrag beinhaltet keine Studien an Menschen oder Tieren.

\section{Literatur}

1. Allan CK (2011) Intensive care of the adult patient with congenital heart disease. Prog Cardiovasc Dis 53:274280

2. Ammash N, Warnes CA (1996) Cerebrovascular events in adult patients with cyanotic congenital heart disease. J Am Coll Cardiol 28:768-772

3. Ammash NM, Connolly HM, Abel MD et al (1999) Noncardiac surgery in eisenmenger syndrome. J Am Coll Cardiol 33:222-227

4. Atallah J, Erickson CC, Cecchin F et al (2013) Multi-institutional study of implantable defibrillator lead performance in children and young adults: results of the Pediatric Lead Extractability and Survival Evaluation (PLEASE) Study. Circulation 127:2393-2402

5. Bae EJ, Noh Cl, Choi JY et al (2005) Late occurrence of adenosine-sensitive focal junctional tachycardia in complex congenital heart disease. J Interv Card Electrophysiol 12:115-122

6. Berdat PA, Immer F, Pfammatter JP et al (2004) Reoperations in adults with congenital heart disease: analysis of early outcome. Int J Cardiol 93:239-245

7. Burrows FA (1989) Physiologic dead space, venous admixture, and the arterial to end-tidal carbon dioxide difference in infants and children undergoing cardiac surgery. Anesthesiology 70:219-225

8. Cannesson M, Earing MG, Collange V et al (2009) Anesthesia for noncardiac surgery in adults with congenital heart disease. Anesthesiology 111:432-440

9. Chassot PG, Bettex DA (2006) Anesthesia and adult congenital heart disease. J Cardiothorac Vasc Anesth 20:414-437

10. Chun DS, Schamberger MS, Flaspohler T et al (2004) Incidence, outcome, and risk factors for stroke after the Fontan procedure. Am J Cardiol 93:117-119

11. Daliento L, Somerville J, Presbitero P et al (1998) Eisenmenger syndrome. Factors relating to deterioration and death. Eur Heart J 19:1845-1855

12. Davlouros PA, Niwa K, Webb G et al (2006) The right ventricle in congenital heart disease. Heart 92(Suppl 1):i27-i38 
13. Deanfield J, Thaulow E, Warnes C et al (2003) Management of grown up congenital heart disease. Eur Heart J 24:1035-1084

14. Dimopoulos K, Diller GP, Koltsida E et al (2008) Prevalence, predictors, and prognostic value of renal dysfunction in adults with congenital heart disease. Circulation 117:2320-2328

15. Dore A, Glancy DL, Stone $S$ et al (1997) Cardiac surgery for grown-up congenital heart patients: survey of 307 consecutive operations from 1991 to 1994. Am J Cardiol 80:906-913

16. Engelfriet $P$, Boersma $E$, Oechslin $E$ et al (2005) The spectrum of adult congenital heart disease in Europe: morbidity and mortality in a 5 year follow-up period. The Euro Heart Survey on adult congenital heart disease. Eur Heart J 26:2325-2333

17. Engelfriet PM, Duffels MG, Moller T et al (2007) Pulmonary arterial hypertension in adults born with a heart septal defect: the Euro Heart Survey on adult congenital heart disease. Heart 93:682-687

18. Epstein AE, Dimarco JP, Ellenbogen KA et al (2008) ACC/AHA/HRS 2008 guidelines for device-based therapy of cardiac rhythm abnormalities: a report of the American College of Cardiology/American Heart Association Task Force on Practice Guidelines (Writing Committee to revise the ACC/AHA/NASPE 2002 guideline update for implantation of cardiac pacemakers and antiarrhythmia devices) developed in collaboration with the American Association for Thoracic Surgery and Society of Thoracic Surgeons. J Am Coll Cardiol 51:e1-e62

19. Flanagan MF, Hourihan M, Keane JF (1991) Incidence of renal dysfunction in adults with cyanotic congenital heart disease. Am J Cardiol 68:403-406

20. Foster E, Graham TP Jr, Driscoll DJ et al (2001) Task force 2: special health care needs of adults with congenital heart disease. J Am Coll Cardiol 37:1176-1183

21. Franklin WJ, Gandhi M (2012) Congenital heart disease in pregnancy. Cardiol Clin 30:383-394

22. Gatzoulis MA, Balaji S, Webber SA et al (2000) Risk factors for arrhythmia and sudden cardiac death late after repair of tetralogy of Fallot: a multicentre study. Lancet 356:975-981

23. Giamberti A, Chessa M, Abella R et al (2009) Morbidity and mortality risk factors in adults with congenital heart disease undergoing cardiac reoperations. Ann Tho rac Surg 88:1284-1289

24. Habib G, Hoen B, Tornos P et al (2009) Guidelines on the prevention, diagnosis, and treatment of infective endocarditis (new version 2009): the Task Force on the Prevention, Diagnosis, and Treatment of Infective Endocarditis of the European Society of Cardiology (ESC). Endorsed by the European Society of Clinical Microbiology and Infectious Diseases (ESCMID) and the International Society of Chemotherapy (ISC) for Infection and Cancer. Eur Heart J 30:2369-2413

25. Hosking MP, Beynen FM (1992) The modified Fontan procedure: physiology and anesthetic implications. Cardiothorac Vasc Anesth 6:465-475

26. Kaemmerer H, Fratz S, Bauer U et al (2003) Emergency hospital admissions and three-year survival of adults with and without cardiovascular surgery for congenital cardiac disease. J Thorac Cardiovasc Surg 126:1048-1052

27. Kannankeril PJ, Anderson ME, Rottman JN et al (2003) Frequency of late recurrence of intra-atrial reentry tachycardia after radiofrequency catheter ablation in patients with congenital heart disease. Am J Cardiol 92:879-881

28. Khairy P, Landzberg MJ (2008) Adult congenital heart disease: toward prospective risk assessment of a multisystemic condition. Circulation 117:2311-2312

29. Kleemann T, Becker T, Doenges K et al (2007) Annual rate of transvenous defibrillation lead defects in implantable cardioverter-defibrillators over a period of $>10$ years. Circulation 115:2474-2480
30. Kopf GS, Laks H, Stansel HC et al (1990) Thirty-year follow-up of superior vena cava-pulmonary artery (Glenn) shunts. J Thorac Cardiovasc Surg 100:662-670 discussion 670-661)

31. Landzberg MJ, Murphy DJ Jr, Davidson WR Jr et al (2001) Task force 4: organization of delivery systems for adults with congenital heart disease. J Am Coll Cardiol 37:1187-1193

32. Li W, Somerville J (2000) Atrial flutter in grown-up congenital heart (GUCH) patients. Clinical characteristics of affected population. Int J Cardiol 75:129-137 (discussion 138-129)

33. Li W, Somerville J (1998) Infective endocarditis in the grown-up congenital heart (GUCH) population. Eur Heart J 19:166-173

34. Lindberg L, Olsson AK, Jogi P et al (2002) How common is severe pulmonary hypertension after pediatric cardiac surgery? J Thorac Cardiovasc Surg 123:11551163

35. Lovell AT (2004) Anaesthetic implications of grown-up congenital heart disease. Br J Anaesth 93:129-139

36. Lowe BS, Therrien J, lonescu-Ittu R et al (2011) Diagnosis of pulmonary hypertension in the congenital heart disease adult population impact on outcomes. J Am Coll Cardiol 58:538-546

37. Mackie AS, Pilote L, lonescu-Ittu R et al (2007) Health care resource utilization in adults with congenital heart disease. Am J Cardiol 99:839-843

38. Macmahon B, Mckeown T, Record RG (1953) The incidence and life expectation of children with congenital heart disease. Br Heart J 15:121-129

39. Mahajan A, Shabanie A, Turner J et al (2003) Pulse contour analysis for cardiac output monitoring in cardiac surgery for congenital heart disease. Anesth Analg 97:1283-1288

40. Marelli AJ, Mackie AS, lonescu-Ittu R et al (2007) Congenital heart disease in the general population: changing prevalence and age distribution. Circulation 115:163-172

41. Martinez-Quintana E, Rodriguez-Gonzalez F, FabregasBrouard M et al (2009) Serum and 24-hour urine analysis in adult cyanotic and noncyanotic congenital heart disease patients. Congenit Heart Dis 4:147-152

42. Mchugh KE, Hillman DG, Gurka MJ et al (2010) Threestage palliation of hypoplastic left heart syndrome in the University HealthSystem Consortium. Congenit Heart Dis 5:8-15

43. Oechslin E (2004) Hematological management of the cyanotic adult with congenital heart disease. Int J Cardiol 97(Suppl 1):109-115

44. Oechslin EN, Harrison DA, Connelly MS et al (2000) Mode of death in adults with congenital heart disease. Am J Cardiol 86:1111-1116

45. Paul T, Bertram H, Kriebel T et al (2000) Supraventricular tachycardia in infants, children and adolescents: diagnosis, drug and interventional therapy. Z Kardiol 89:546-558

46. Pflaumer A, Chard R, Davis AM (2012) Perspectives in interventional electrophysiology in children and those with congenital heart disease: electrophysiology in children. Heart Lung Circ 21:413-420

47. Price LC, Wort SJ, Finney SJ et al (2010) Pulmonary vascular and right ventricular dysfunction in adult critical care: current and emerging options for management: a systematic literature review. Crit Care 14:R169

48. Price S, Jaggar SI, Jordan S et al (2007) Adult congenital heart disease: intensive care management and outcome prediction. Intensive Care Med 33:652-659

49. Rauch $\mathrm{H}$, Muller M, Fleischer F et al (2002) Pulse contour analysis versus thermodilution in cardiac surgery patients. Acta Anaesthesiol Scand 46:424-429

50. Report of the British Cardiac Society Working P (2002) Grown-up congenital heart (GUCH) disease: current needs and provision of service for adolescents and adults with congenital heart disease in the UK. Heart 88(Suppl 1):i1-i14
51. Rigolin VH, Li JS, Hanson MW et al (1997) Role of right ventricular and pulmonary functional abnormalities in limiting exercise capacity in adults with congenital heart disease. Am J Cardiol 80:315-322

52. Rudiger A, Bosshart M, Breitenstein A et al (2012) Die akute Rechtsherzinsuffizienz. Teil 2: Therapie. Schweiz Med Forum 13:364-368

53. Rudiger A, Breitenstein A, Bosshart M et al (2012) Die akute Rechtsherzinsuffizienz. Teil 1:Mechanismen und Diagnostik. Schweiz Med Forum 12:347-351

54. Schummer W, Schummer C, Frober R (2003) Persistent left superior vena cava and central venous catheter position: clinical impact illustrated by four cases. Surg Radiol Anat 25:315-321

55. Seal R (2011) Adult congenital heart disease. Paediatr Anaesth 21:615-622

56. Shah H, Kuehl K, Sherker AH (2010) Liver disease after the Fontan procedure: what the hepatologist needs to know. J Clin Gastroenterol 44:428-431

57. Siu SC, Sermer M, Colman JM et al (2001) Prospective multicenter study of pregnancy outcomes in women with heart disease. Circulation 104:515-521

58. Somerville J (2001) Grown-up congenital heart disease - medical demands look back, look forward 2000. Thorac Cardiovasc Surg 49:21-26

59. Somerville J (1997) Management of adults with congenital heart disease: an increasing problem. Annu Rev Med 48:283-293

60. Tempe DK, Virmani S (2002) Coagulation abnormalities in patients with cyanotic congenital heart disease. J Cardiothorac Vasc Anesth 16:752-765

61. Thorne SA, Barnes I, Cullinan P et al (1999) Amiodarone-associated thyroid dysfunction: risk factors in adults with congenital heart disease. Circulation 100:149-154

62. Van Nieuwenhuizen RC, Peters $M$, Lubbers $L$ et al (1999) Abnormalities in liver function and coagulation profile following the Fontan procedure. Heart 82:40-

63. Verheugt $\mathrm{CL}$, Uiterwaal CS, Van Der Velde ET et al (2010) The emerging burden of hospital admissions of adults with congenital heart disease. Heart 96:872878

64. Warnes CA (2009) Adult congenital heart disease importance of the right ventricle. J Am Coll Cardio 54:1903-1910

65. Warnes CA, Liberthson R, Danielson GK et al (2001) Task force 1: the changing profile of congenital heart disease in adult life. J Am Coll Cardiol 37:1170-1175

66. Warnes CA, Williams RG, Bashore TM et al (2008) ACC/ AHA 2008 guidelines for the management of adults with congenital heart disease: a report of the American College of Cardiology/American Heart Association Task Force on Practice Guidelines (Writing Committee to develop guidelines on the management of adults with congenital heart disease). Developed in collaboration with the American Society of Echocardiography, Heart Rhythm Society, International Society for Adult Congenital Heart Disease, Society for Cardiovascular Angiography and Interventions, and Society of Thoracic Surgeons. J Am Coll Cardiol 52:e143-e263

67. Wood $\mathrm{P}$ (1958) The Eisenmenger syndrome or pulmonary hypertension with reversed central shunt. Br Med J 2:755-762 